\title{
ANLN Promotes Carcinogenesis in Oral Cancer by Regulating PI3K/mTOR Signaling Pathway
}

\section{Bing Wang}

Xinjiang Medical University Affiliated First Hospital

\section{Xiaoli Zhang}

People's hospital of Xinjiang Uygur autonomous region

\section{Chenxi Li}

University Hospital Hamburg-Eppendorf (UKE)

\section{Ningning Liu}

Xinjiang medical university affilliated first hospital

\section{Min $\mathrm{Hu}$}

Urumqi Myour dental clinic

\section{Zhongcheng Gong ( $\nabla$ gump0904@126.com )}

Oncological Department of Oral and Maxillofacial Surgery, The First Affiliated Hospital of Xinjiang Medical University, Stomatology School of Xinjiang Medical University, Stomatology Research Institute of Xinjiang Autonomous Region. Urumqi 830054, China.

\section{Research}

Keywords: ANLN, Oral cancer, mTOR, PI3K signaling pathway

Posted Date: October 26th, 2020

DOI: https://doi.org/10.21203/rs.3.rs-95506/v1

License: (c) (i) This work is licensed under a Creative Commons Attribution 4.0 International License. Read Full License 


\section{Abstract}

Background: Oral cancer is a malignant disease threatening human's life and greatly reduces human's life quality. ANLN was reported to promote progression in cancer. This study aims at investigating the role of ANLN and the molecular mechanism in oral cancer.

Methods: ANLN was down-regulated by RNAi technology. The effect of ANLN on cell behaviors including proliferation, cycle distribution, invasion, and apoptosis was detected. Western blotting analysis was used to explore the mechanism of ANLN in oral cancer.

Results: Based on data from TCGA database, ANLN was shown to be expressed significantly higher in tumor tissues than the normal control. Patients with higher ANLN expression exhibited shorter survival time. ANLN was also expressed abundantly in cancer cell lines CAL27 and HN30. When ANLN was knocked down in CAL27 and HN30 cells, cell proliferation and colony formation ability was downregulated. Cell invasion ability was also suppressed. But cell apoptosis rate was induced reversely. In addition, the level of critical members in PI3K signaling pathway including PI3K, mTOR, Akt, and PDK-1 were significantly reduced after ANLN was knocked down in CAL27 cells.

Conclusions: ANLN contributes to progression in oral cancer and affects activation of $\mathrm{PI} / \mathrm{K} / \mathrm{mTOR}$ signaling pathway. This study provides a new potential target for therapy and drug development in oral cancer.

\section{Background}

Actin is an important molecule responsible for cell motility and contraction of muscle cells. Anillin actin binding protein (ANLN) is an actin-binding protein and plays critical roles in cell growth, cell migration, and cytoskeletal dynamics in podocytes [1]. ANLN gene is mapped at location 7p14.2 in human chromosome. Mutations in ANLN are detected in focal segmental glomerulosclerosis and more experiments suggest that ANLN contributes to tumor progression [2]. For example, over-expression of ANLN promoted cell metastasis in lung adenocarcinoma and patients with higher ANLN expression displayed much worse prognosis [3, 4]. Over-expression of ANLN was found in pancreatic cancer and down-regulation of ANLN by microRNA inhibited cell aggressiveness ability $[5,6]$. In cervical cancer, bioinformatics analysis demonstrated that ANLN might be a candidate biomarker for evaluation of survival [7]. Meanwhile, ANLN increased chemotherapy resistance ability of breast cancer and reduced the effectiveness of Doxorubicin or Anthracycline in clinic [8, 9]. Based on large published profiles, ANLN promotes progression in cancer and might be a novel target in cancer therapy [10].

Oral cancer is a subtype of head and neck squamous cell carcinoma, accounting for about $2 \%$ of all cancer patients and the death rate is about $50 \%$ [11]. Patients with localized disease could benefit from contemporary therapies including surgery, chemotherapy and radiotherapy. But the prognosis is dismal for those with metastatic disease. And tumor stage of patients is a great factor affecting survival rate. The overall 5 -year survival rate is about $80 \%$ for stage I patients but $40 \%$ for stage $\otimes$ patients $[11,12]$. 
Moreover, the quality of patient's life is down-regulated greatly due to the malfunction of talking, cosmetic appearance and eating [13]. Therefore, it is urgent to disclose the pathogeny of oral cancer and the underlying molecular mechanism.

ANLN was reported to promote progression in a series of cancers, but its function in oral cancer is not known. Shimizu reported that the mRNA level of ANLN gene was up-regulated in head and neck squamous cell carcinoma [14]. However, no further information was published. In this study, to investigate the role of ANLN in oral cancer, we reduced the expression of ANLN in oral cancer cells and determined the ability of cell proliferation, aggressiveness, cell apoptosis, and cell cycle in oral cancer. In addition, the signaling pathway regulated by ANLN was preliminarily explored.

\section{Methods}

\section{Cell lines and cell culture}

Human oral cancer cell lines HN30 and CAL27 were bought from Chinese Academy of Sciences (Shanghai, China) and cultured in DMEM medium (Gibco, CA, USA) containing 10\% FBS (Gibco, CA, USA) and $1 \%$ penicillin-streptomycin (Beyotime, Shanghai, China).

\section{Synthesis of small interfere RNA oligonucleotides and transfection}

Two small RNA oligonucleotide fragments (siRNA-1\#, siRNA-2\#) targeting human ANLN gene were designed and synthesized. The target sequence for human ANLN gene was listed in Table 1. Then Lipo3000 reagent was used to transfer the two siRNA into oral cancer cells. In brief, $1 \mu \mathrm{L}$ of Lipo3000 (Invitrogen, CA, USA) was mixed with 50 pmol siRNA for $20 \mathrm{~min}$ at room temperature. Then the mix was added into prepared cells in 24-well plates. After culture for $6 \mathrm{~h}$, the supernatant was removed and new fresh medium was added. Then cells were cultured for another $48 \mathrm{~h}$.

\section{Realtime quantitative polymerase chain reaction assay (qPCR)}

Total RNA was extracted from cultured cells with RNA easy Kit (Beyotime, Shanghai, China) according to the manufacturer's instruction. Briefly, cells were lysed with lysis buffer and binding buffer was added into the lysis mix. Then the mixture was transferred into collection column and washed with washing buffer A/B. At last, RNA was eluted with elution buffer and quantified on an ultraviolet spectrophotometer (Onedrop1000, Hangzhou, China).

About $0.5 \mu \mathrm{g}$ of total RNA was used to synthesize first chain of cDNA with cDNA synthesize kit (Yeasen, Shanghai, China). Then $1 \mu \mathrm{L}$ cDNA was used for qPCR assay with SYBR GREEN mix (Beyotime, Shanghai, China) on ABI 7000 system (Applied Biosystems, USA). The protocol for qPCR assay was as following: $95^{\circ} \mathrm{C}, 5 \mathrm{~min}$; $\left(95^{\circ} \mathrm{C}, 10 \mathrm{sec} ; 60^{\circ} \mathrm{C}, 15 \mathrm{sec}\right)$ for 40 cycles. b-actin was chosen as internal control. The relative mRNA expression level of each gene was calculated by $2^{-\triangle \triangle C T}$ method. The primers were showed in Table 2. 


\section{Western blotting assay}

Total protein from cultured cells was prepared with Protein-extract kit (Beyotime, Shanghai, China) according to the manufacturer's instruction. In brief, cells were collected by centrifugation at $1200 \mathrm{rpm}$ for $5 \mathrm{~min}$ at room temperature. The cell body was resuspended with lysis buffer and transferred into the protein column and washed with serial buffer. At last, protein was eluted with elution buffer and the concentration of total protein was detected on an ultraviolet spectrophotometer (Onedrop1000, Hangzhou, China).

$10 \mu \mathrm{g}$ total protein was separated on $15 \%$ SDS-PAGE GEL for $1.5 \mathrm{~h}$. Then candidate protein was transferred onto PVDF membranes (Beyotime, Shanghai, China) and analyzed by western blotting analysis. In brief, the PVDF membranes were blocked with $1 \%$ BSA for $1 \mathrm{~h}$ at room temperature and washed with PBS for three times. Then, primary antibody against each candidate protein was co-cultured with PVDF membrane for $12 \mathrm{~h}$ at $4^{\circ} \mathrm{C}$. After washed with PBS for three times, secondary antibody was cocultured with the PVDF membranes for $2 \mathrm{~h}$ at room temperature. At last, after the PVDF membranes were washed with PBS, each candidate protein was detected by ECL kit (Yeasen, Shanghai, China) according to the manufacturer's instructions. The information of primary antibodies were as following: PI3K (ab140307, 1:2000, Abcam, CA, USA), mTOR (ab134903, 1:6000, Abcam, CA, USA), AKT (ab18785, 1:1000, Abcam, CA, USA), PDK-1 (ab207450, 1:2000, Abcam, CA, USA), GAPDH (ab9485, 1:2000, Abcam, CA, USA).

\section{Cell proliferation assay}

Oral cancer cells treated with siRNA were seeded into a 96 -well plate at $5 \times 10^{3} / 100 \mu \mathrm{L} /$ well and cultured for $96 \mathrm{~h}$. Then $10 \%$ of total volume of CCK-8 reagent was added into each well every $24 \mathrm{~h}$ and cultured for another $1 \mathrm{~h}$. The absorbance value at $450 \mathrm{~nm}$ wavelength was detected on a microplate reader (Tecan, Switzerland). Each experiment was repeated independently for three times.

\section{Plate colony formation}

Oral cancer cells treated with siRNA were seeded into a 24 -well plate at $0.5 \times 10^{3} / 100 \mu \mathrm{L} /$ well and cultured for 14 days. After 14 days, cells were fixed in $4 \%$ paraformaldehyde (Beyotime, Shanghai, China) for 15 min and washed with PBS for three times. Then cells were stained in $0.5 \%$ crystal staining buffer (Sangon, Shanghai, China) for $15 \mathrm{~min}$ at room temperature. After washed with PBS for three times, positively stained cells were calculated under a microscope (OLYMPUS, Tokyo, Japan). Each experiment was repeated independently for three times.

\section{Transwell assay}

Transwell insert with $8 \mu \mathrm{m}$ pore was treated with $200 \mu \mathrm{L}$ matrigel (BD science, USA) and placed overnight. After washed with $200 \mu \mathrm{L}$ culture medium, oral cancer cells treated with siRNA were seeded into the insert at $1 \times 10^{4} / 300 \mu \mathrm{L} /$ well and placed into a 24 -well plate. Culture medium with no FBS was added into the 
upper room of the insert when culture medium with $10 \%$ FBS was added into the lower well of the 24-well plate. After cultured for $48 \mathrm{~h}$, cells in the upper layer of the insert were scraped gently and cells in the lower layer of the insert were fixed in $4 \%$ paraformaldehyde (Beyotime, Shanghai, China) for $15 \mathrm{~min}$ and washed with PBS for three times. Then cells were stained in $0.5 \%$ crystal staining buffer (Sangon, Shanghai, China) for 15 min at room temperature. After washed with PBS for three times, positively stained cells were calculated under a microscope (OLYMPUS, Tokyo, Japan). Each experiment was repeated independently for three times.

\section{Cell apoptosis detection}

Oral cancer cells treated with siRNA were seeded into a 6 -well plate at $2 \times 10^{5} / 500 \mu \mathrm{L} /$ well and culture for $48 \mathrm{~h}$. Then cells were treated with Apoptosis-detecton-kit (Beyotime, Shanghai, China). In brief, cells were collected by centrifugation at $1200 \mathrm{rpm}$ for $5 \mathrm{~min}$ at room temperature. Then cells were resuspended in $100 \mu \mathrm{L}$ staining buffer containing $5 \mu \mathrm{L}$ Annexin V/FITC and 10 $\mathrm{L}$ PI reagent. After culture for $15 \mathrm{~min}$ at room temperature in the dark, $400 \mu \mathrm{L}$ staining buffer was added and cell apoptosis was analyzed on a flow cytometer (BD, FACSCelesta, CA, USA). Each experiment was repeated independently for three times.

\section{Cell cycle detection}

Oral cancer cells treated with siRNA were seeded into a 6 -well plate at $2 \times 10^{5} / 500 \mu \mathrm{L} /$ well and culture for $48 \mathrm{~h}$. Then cells were treated with Cell cycle-detecton-kit (Yeasen, Shanghai, China). In brief, cells were collected by centrifugation at $1200 \mathrm{rpm}$ for $5 \mathrm{~min}$ at room temperature. Then cells were resuspended in $500 \mu \mathrm{L}$ staining buffer containing $10 \mu \mathrm{L}$ RNase A and 10 $\mathrm{L} \mathrm{PI}$ reagent. After culture for $30 \mathrm{~min}$ at room temperature in the dark, cell cycle distribution was analyzed on a flow cytometer (BD, FACSCelesta, CA, USA). Each experiment was repeated independently for three times.

\section{Statistical analysis}

All statistical analysis was carried out on SPSS 16.0 software. All data was displayed as mean value plus standard variation (mean \pm sd). In brief, the difference between two groups was analyzed by unpaired student't T test. One-way ANOVA analysis was used to analyze the difference among multiple groups. $P$ value less than 0.05 was considered as statistically significant.

\section{Results}

\section{ANLN is associated with survival in patients with oral cancer}

To evaluate the clinical significance of ANLN in oral cancer, the expression data of ANLN and clinical profile were downloaded from TCGA database. As shown in Figure 1A, ANLN was expressed significantly higher in tumor tissues than the adjacent normal control tissues. The 5-year survival rate of patients with high ANLN expression was much worse than the patients with low ANLN expression (p囚0.05) (Figure 1B). 
In addition, ANLN was expressed abundantly in tumor cell lines CAL27 and HN30 (Figure 1C). These data suggest that ANLN is important in oral cancer.

\section{ANLN contributes to cell growth and proliferation}

To investigate the role of ANLN in oral cancer, the expression of ANLN in CAL27 and HN30 was reduced by RNAi technology. The mRNA level of ANLN was reduced by $80 \%$ in CAL27 and $72.3 \%$ in HN30 cells (Figure 2A and 2B). Then cell proliferation rate in CAL27 was decreased by $59.3 \%$ when it was $44 \%$ in HN30 cells (Figure 2C and 2D). The colony formation ability was reduced by $67.7 \%$ in CAL27 cells while $45 \%$ in HN30 cells (Figure 2E and 2F). So, ANLN contributes to cell growth and proliferation in oral cancer.

\section{ANLN is not necessary to cell cycle transition}

PI staining was used to detect cell cycle distribution in cells with ANLN knockdown. As shown in Figure $3 \mathrm{~A}$ and $3 \mathrm{~B}$, cells in $\mathrm{G} 1$ phase increased slightly when ANLN was reduced in CAL27 cells. In HN30 cells, the ratio in $\mathrm{G} 1$ phase also increased a little (Figure 3C and 3D). In both two cell lines, ANLN knockdown blocked cell cycle transition from $\mathrm{G} 1$ phase to $S$ phase but not significant, which suggests the little influence of ANLN on cell cycle in oral cancer.

\section{Cell invasion is suppressed by ANLN knockdown}

To detect the effect of ANLN knockdown on invasive ability of oral cancer, transwell assay was designed. As shown in Figure 4A and 4B, the relative cell invasive rate in CAL27 cells was $48.7 \%$ when ANLN was reduced. In consistent, it was $36.4 \%$ in HN30 cells (Figure $4 \mathrm{C}$ and $4 \mathrm{D}$ ). In both cell lines, the invasive ability was suppressed when ANLN was reduced. This suggests that ANLN might be a critical factor in metastasis in oral cancer.

\section{Cell apoptosis is induced by ANLN knockdown}

Resistant to programmed cell death or apoptosis is common in tumor. Double staining with FITC and PI dye was designed to detect cell apoptosis in oral cancer. The cell apoptosis rate increased from $0.88 \%$ to $15.24 \%$ when ANLN was reduced in CAL27 cells (Figure 5A and 5B). Consistently, it increased from $3.72 \%$ to $36.88 \%$ in HN30 cells (Figure $5 \mathrm{C}$ and $5 \mathrm{D}$ ). In conclusion, reduced ANLN expression induced cell apoptosis in both CAL27 and HN30 cells, which suggests that ANLN inhibits cell apoptosis in oral cancer.

\section{$\mathrm{PI3K} / \mathrm{mTOR}$ signaling is activated by ANLN}

$\mathrm{PI} 3 \mathrm{~K} / \mathrm{mTOR}$ signaling plays important role in cell survival and proliferation. In this study, the expression of PI3K, mTOR, and Akt was regulated by ANLN in CAL27 cells. As shown in Figure 6A, reduced ANLN in CAL27 cells decreased the expression level of PI3K, mTOR, PDK1, and Akt. The level of PI3K molecule decreased by $22 \%$ after ANLN was knocked down. It was $54 \%, 52 \%$, and $41 \%$ for mTOR, PDK 1 , and Akt, respectively (Figure 6B). These molecules are key members in PI3K/mTOR signaling. Therefore, ANLN contributes to activation of $\mathrm{PI} 3 \mathrm{~K} / \mathrm{mTOR}$ signaling in oral cancer. 


\section{Discussion}

In the past years, major advances in therapy for oral cancer have been made. Systemic therapy such as chemotherapy and immunotherapy drugs did bring positive improvement for patients with oral cancer $[11,12,15]$. However, damage to normal tissues is a big problem in systemic therapy and will be a great challenge for a very long time. Besides unspecific adverse effects, failure in talking, eating and entertaining caused by oral cancer greatly reduces patient's life quality [13]. According to previous study, the overall survival rate of patients with oral cancer was dependent on tumor stage $[11,12]$. Patients with higher stage displayed worse survival in clinic. Therefore, early diagnosis is critical for therapy and prognosis in oral cancer patients. Unfortunately, oral cancer is a heterogeneous disease, which leads to different responses to a particular treatment [16]. Accordingly, it is conceived that a specific marker in a particular stage will be helpful for therapy in oral cancer.

Based on the large data from Pubmed database, a series of genes have been shown to play critical roles in oral cancer. Advance in bio-technique and medical knowledge is a big factor, but variance in tumor genetics also plays a certain role. It is unveiled that genome instability is a common phenomenon in cancer [17]. In each cancer, several dozens of gene variance could be detected out. In a particular cohort of patients, one or two gene variance might dominate the progression or metastasis. ANLN is an actinbinding protein responsible for cell motility [1]. Multiple research stated that ANLN was associated with poor survival in cancer patients [2]. In this study, we analyzed the data of ANLN in TCGA database and found that ANLN was over-expressed in oral cancer specimens compared to the adjacent normal control. And higher ANLN expression was associated with shorter survival time. This data further supports that ANLN is associated with poor prognosis in cancer patients and enlarges the list of cancers affected by ANLN.

Based on the public data, ANLN could promote cell proliferation and growth in cancer and increase the ability of cell migration and invasion [2-7]. Our data was consistent with previous study and for first time, we disclosed the secret of ANLN in oral cancer. ANLN was essential to cell growth, proliferation, and invasion in oral cancer. These traits are the same as the typical hallmarks of cancer. Unlimited expansion and growth without contact-inhibition is a great advantage of cancer cells [18]. Cancer cells could keep expansion if only the energy is enough. Distant metastasis is the end of a cancer patient. In cancer, cells could flow out of the primary loci by invading the surrounded vasculature or by lymphatic vessels [19-21]. It is published that tumor cells could secret matrix metal protease to melt the extracellular matrix and alter the permeability of blood vessels, which leads to leakage of blood vessels. This is an important mechanism and a list of drugs improving blood vessel leakage is being developed [22, 23]. In this study, knockdown of ANLN suppressed cell invasion in oral cancer, which suggests that ANLN might be a new target for drug development. Resistant to apoptosis is evolutionarily reserved by tumor. But this mechanism is employed by chemo-radiation therapy [24, 25]. In clinic, chemotherapy drugs could directly causes cell apoptosis or increases the sensitivity of tumor cells to radiation. ANLN displayed antagonistic activity to apoptosis in this study. This suggests that knockdown or knockout of ANLN might be a good way to induce cell apoptosis in oral cancer. 
A complete life cycle of cells includes G1, S, G2, and M phase. In cancer, this cycle is shortened and cell division is accelerated. This leads to quick increase of cell number in a short time [26, 27]. In this study, knockdown of ANLN didn't block transition of G1 phase to $S$ phase significantly in oral cancer. It is possible that ANLN might not be a checkpoint controlling cell cycle in oral cancer.

In all, the above data shows that ANLN plays promoting role in oral cancer. But what's the molecular mechanism of ANLN in oral cancer? The present data demonstrated that signaling molecules including PI3K, PDK1, Akt and mTOR were regulated by ANLN in oral cancer. These molecules are critical members in $\mathrm{PI} 3 \mathrm{~K} / \mathrm{mTOR}$ signaling pathway. PI3K/mTOR signaling is an important signaling in development and homeostasis [28]. Abnormal activation of PI3K/mTOR signaling often causes serious disease especially cancer $[28,29]$. In cancer, activation of PI3K/mTOR signaling results in cell proliferation, prolongs cell survival, shortens cell cycle and suppressed cell apoptosis. For example, PI3K/mTOR signaling was activated in lung cancer and drug targeting this signaling was being investigated [30]. In liver cancer, cell apoptosis was inhibited through activation of PI3K/mTOR signaling [31]. In oral cancer, PI3K/mTOR signaling also contributes to progression and deterioration. Inhibitors against PI3K/mTOR signaling could increase radiosensitization of oral cancer cells [32,33]. What's funny, ANLN exerted influence on $\mathrm{PI} 3 \mathrm{~K} / \mathrm{mTOR}$ signaling in previous research. Mutation in ANLN resulted in dysregulated $\mathrm{PI} 3 \mathrm{~K} / \mathrm{mTOR}$ signaling in podocytes [1]. ANLN regulated PI3K/Akt signaling in lung cancer and promoted cancer progression [34]. In this study, our data points to that ANLN could also affect activation of PI3K/mTOR signaling in oral cancer. This enlarges our knowledge about the role and mechanism of ANLN in oral cancer. However, more experiments are necessary to bridge the link of ANLN and PI3K/mTOR signaling. And the clinical meaning of ANLN in a large cohort of oral cancer specimen will further support the data from TCGA database. In addition, the in vivo role of ANLN in xenograft mice model is the follow-up.

\section{Conclusion}

In summary, ANLN was overexpressed and associated with patient's prognosis in oral cancer. ANLN regulated cell growth, proliferation, invasion, and apoptosis in vitro. ANLN participated in activation of $\mathrm{PI} 3 \mathrm{~K} / \mathrm{mTOR}$ signaling. Patients with oral cancer will benefit from this study in a day.

\section{Abbreviations}

ANLN: anillin actin binding protein

mTOR: mammalian target of rapamycin

TCGA: The cancer genome atlas

DMEM: Dulbecco's modification of Eagle's medium

QPCR: Realtime quantitative polymerase chain reaction assay 
ECL: Efficient chemiluminescence kit

CCK-8: Cell counting kit-8

RNAi: RNA interference

\section{Declarations}

Ethics approval and consent to participate

Not applicable.

\section{Consent for publication}

Not applicable.

\section{Availability of data and materials}

The datasets used and/or analyzed during the current study are available from the corresponding author on reasonable request.

\section{Competing interests}

The authors declare that they have no competing interests.

\section{Funding}

This study was supported by National Natural Science Foundation of China (grant number: 81760191) and Xinjiang Special Scientific Research Project of Youth Medical Science and Technology (WJWY201924).

\section{Author's contributions}

CXL and ZCG designed the study, reviewed the manuscript and guaranteed the integrity of the whole study. BW and XLZ carried out the whole experiments and drafted the manuscript. NNL and MH analyzed the data and literature reports.

\section{Acknowledgement}

Not applicable.

\section{References}

1. Hall G, Lane BM, Khan K, et al. The Human FSGS-Causing ANLN R431C Mutation Induces Dysregulated PI3K/AKT/mTOR/Rac1 Signaling in Podocytes. J Am Soc Nephrol. 2018; 29(8):2110- 
2122.

2. Gbadegesin RA, Hall G, Adeyemo A, et al. Mutations in the Gene That Encodes the F-actin Binding Protein Anillin Cause FSGS. J Am Soc Nephrol. 2014; 25(9):1991-2002.

3. Xu J, Zheng H, Yuan S, et al. Overexpression of ANLN in Lung Adenocarcinoma Is Associated With Metastasis. Thorac Cancer. 2019; 10(8):1702-1709.

4. Long XY, Zhou W, Wang YX, Liu SQ. Prognostic Significance of ANLN in Lung Adenocarcinoma. Oncol Lett. 2018; 16(2):1835-1840.

5. Idichi T, Seki N, Kurahara H, et al. Regulation of Actin-Binding Protein ANLN by Antitumor miR217 Inhibits Cancer Cell Aggressiveness in Pancreatic Ductal Adenocarcinoma. Oncotarget. 2017; 8(32):53180-53193.

6. Wang AB, Dai HS, Gong Y, et al. ANLN-induced EZH2 Upregulation Promotes Pancreatic Cancer Progression by Mediating miR-218-5p/LASP1 Signaling Axis. J Exp Clin Cancer Res. 2019; 38(1):347.

7. Xia LL, Su XL, Shen JZ, et al. ANLNFunctions as a Key Candidate Gene in Cervical Cancer as Determined by Integrated Bioinformatic Analysis. Cancer Manag Res. 2018; 10:663-670.

8. Zhang M, Wang F, Xiang Z, et al. LncRNA XIST Promotes Chemoresistance of Breast Cancer Cells to Doxorubicin by Sponging miR-200c-3p to Upregulate ANLN. Clin Exp Pharmacol Physiol. 2020; doi: 10.1111/1440-1681.13307.

9. Dai XF, Mei Y, Chen X, Cai DY. ANLN and KDR Are Jointly Prognostic of Breast Cancer Survival and Can Be Modulated for Triple Negative Breast Cancer Control. Front Genet. 2019; 10:790.

10. Minh TN, Lee CH. Role of Anillin in Tumour: From a Prognostic Biomarker to a Novel Target. Cancers. 2020; $12(6): 1600$.

11. Li CC, Shen Z, Bavarian R, et al. Oran cancer Genetics and the role of precision medicine. Dent Clin N Am. 2018; 62:29-46.

12. Hartner L. Chemotherapy for oral cancer. Dent Clin North Am. 2018; 62(1):87-97.

13. Valdez JA, Brennan MT. Impact of Oral Cancer on Quality of Life. Dent Clin North Am. 2018; 62(1):143-154.

14. Shimizu S, Seki N, Sugimoto T, et al. Identification of Molecular Targets in Head and Neck Squamous Cell Carcinomas Based on Genome-Wide Gene Expression Profiling. Oncol Rep. 2007; 18(6):1489-97.

15. Sim F, Leidner R, Bell RB. Immunotherapy for head and neck cancer. Oral Maxillofac Surg Clin North Am. 2019; 31(1):85-100.

16. Irimie Al, Ciocan C, Gulei D, et al. Current Insights Into Oral Cancer Epigenetics. Int J Mol Sci. 2018; $19(3): 670$.

17. Jeggo PA, Pearl LH, Carr AM. DNA Repair, Genome Stability and Cancer: A Historical Perspective. Nat Rev Cancer. 2016; 16(1):35-42.

18. O'Neill AC, Alessandrino F, Tirumani SH, Ramaiya NH. Hallmarks of Cancer in the Reading Room: A Guide for Radiologists. AJR Am J Roentgenol. 2018; 211(3):470-484. 
19. Zeeshan R, Mutahir Z. Cancer Metastasis - Tricks of the Trade. Bosn J Basic Med Sci. 2017; 17(3):172-182.

20. Ma QL, Dieterich LC, Detmar M. Multiple Roles of Lymphatic Vessels in Tumor Progression. Curr Opin Immunol. 2018; 53:7-12.

21. Niemiec J, Sas-Korczynska B, Harazin-Lechowska A, et al. Lymphatic and Blood Vessels in Male Breast Cancer. Anticancer Res. 2015; 35(2):1041-8.

22. Koyama S, Matsunaga S, Imanishi M, et al. Tumour Blood Vessel Normalisation by Prolyl Hydroxylase Inhibitor Repaired Sensitivity to Chemotherapy in a Tumour Mouse Model. Sci Rep. 2017; 7:45621.

23. Shen YW, Li ST, Wang X, et al. Tumor Vasculature Remolding by Thalidomide Increases Delivery and Efficacy of Cisplatin. J Exp Clin Cancer Res. 2019; 38(1):427.

24. Mohamed MS, Bishr MK, Almutairi FM, Ali AG. Inhibitors of Apoptosis: Clinical Implications in Cancer. Apoptosis. 2017; 22(12):1487-1509.

25. Pistritto G, Trisciuoglio D, Ceci C, et al. Apoptosis as Anticancer Mechanism: Function and Dysfunction of Its Modulators and Targeted Therapeutic Strategies. Aging (Albany NY). 2016; 8(4):603-19.

26. Shostak A. Circadian Clock, Cell Division, and Cancer: From Molecules to Organism. Int J Mol Sci. 2017; 18(4):873.

27. Ingham M, Schwartz GK. Cell-Cycle Therapeutics Come of Age. J Clin Oncol. 2017; 35(25):29492959.

28. Hillmann P, Fabbro D. PI3K/mTOR Pathway Inhibition: Opportunities in Oncology and Rare Genetic Diseases. Int J Mol Sci. 2019; 20(22):5792.

29. Yang J, Nie J, Ma XL, et al. Targeting PI3K in Cancer: Mechanisms and Advances in Clinical Trials. Mol Cancer. 2019; 18(1):26.

30. Fumarola C, Bonelli MA, Petronini PG, Alfieri RR. Targeting PI3K/AKT/mTOR Pathway in Non Small Cell Lung Cancer. Biochem Pharmacol. 2014; 90(3):197-207.

31. Kim MN, Lee SM, Kim JS, Hwang SG. Preclinical Efficacy of a Novel Dual PI3K/mTOR Inhibitor, CMG002, Alone and in Combination With Sorafenib in Hepatocellular Carcinoma. Cancer Chemother Pharmacol. 2019; 84(4):809-817.

32. Aggarwal S, John S, Sapra L, Sharma S, Das SN. Targeted Disruption of PI3K/Akt/mTOR Signaling Pathway, via PI3K Inhibitors, Promotes Growth Inhibitory Effects in Oral Cancer Cells. Cancer Chemother Pharmacol. 2019; 83(3):451-461.

33. Yu CC, Hung SK, Lin HY, et al. Targeting the PI3K/AKT/mTOR Signaling Pathway as an Effectively Radiosensitizing Strategy for Treating Human Oral Squamous Cell Carcinoma in vitro and in vivo. Oncotarget. 2017; 8(40):68641-68653.

34. Suzuki C, Daigo Y, Ishikawa N, et al. ANLN Plays a Critical Role in Human Lung Carcinogenesis Through the Activation of RHOA and by Involvement in the Phosphoinositide 3-kinase/AKT Pathway. 
Cancer Res. 2005; 65(24):11314-25.

\section{Tables}

Table 1. The target sequence for knockdown of human ANLN gene.

\begin{tabular}{|lll|}
\hline Gene symbol & Name & Target sequence $\left(5^{\prime}-3^{\prime}\right)$ \\
\hline ANLN & SiRNA-1\# & GGACAAAGACACAAGCAAA \\
\cline { 2 - 3 } & SiRNA-2\# & GGAAAAAGAAGAAAAGACA \\
\hline
\end{tabular}

Table 2. Primers for quantification of human ANLN gene.

\begin{tabular}{|llll|}
\hline Gene symbol & Primer & Sequence (5'-3') & Product \\
\cline { 1 - 3 } ANLN & Up & AGTCCTCTGAAAACGGGGGT & 214bp \\
\cline { 2 - 3 } & Down & GTGTGCTACGAGCTGGACTT & \\
\cline { 2 - 4 } & & \multicolumn{2}{|c|}{} \\
\hline
\end{tabular}

\section{Figures}


A

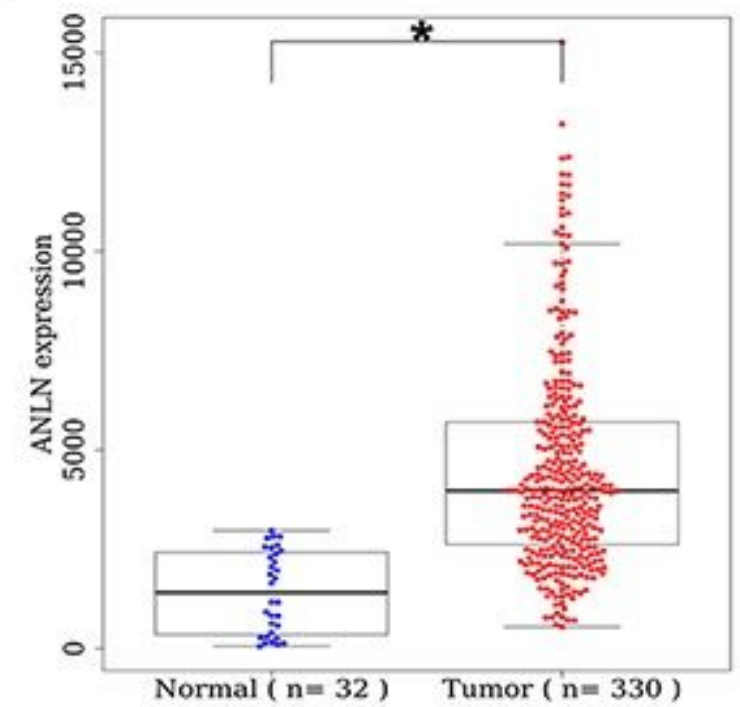

C

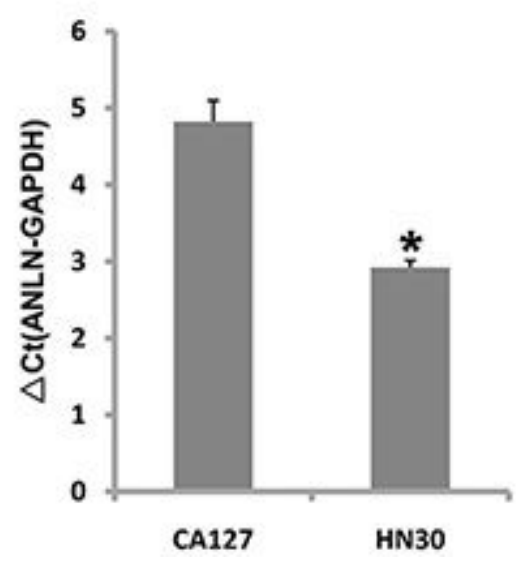

B

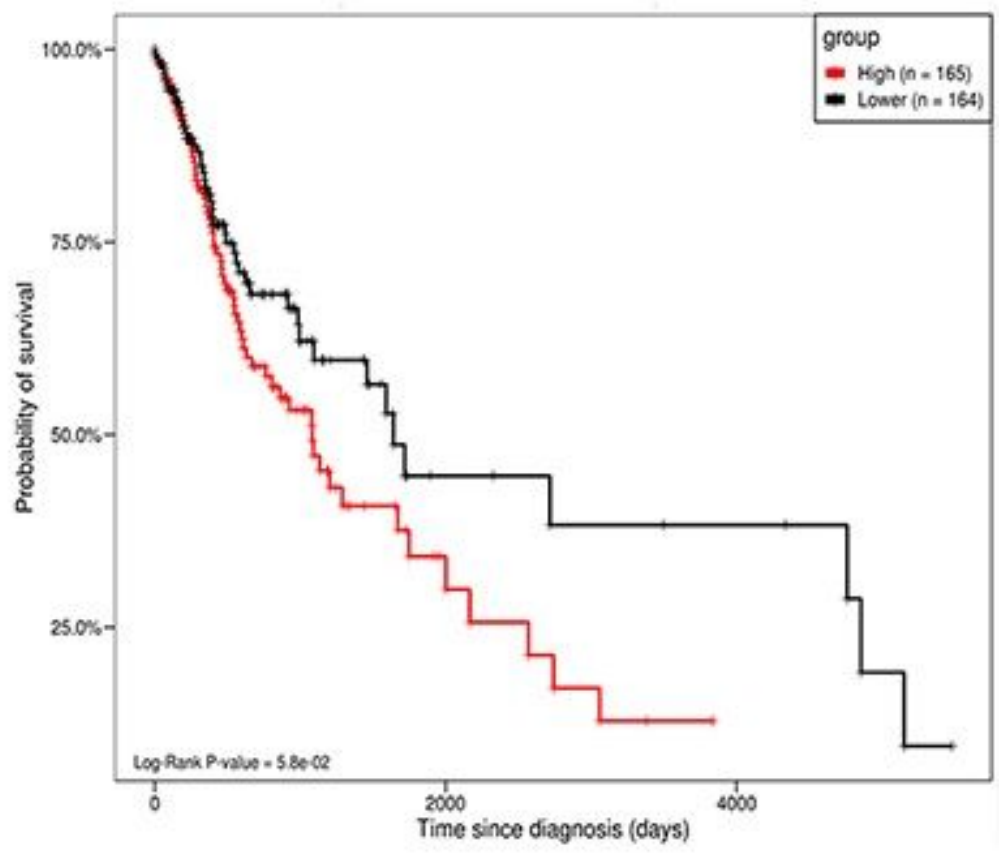

\section{Figure 1}

The expression pattern of ANLN in oral cancer. A. ANLN was overexpressed in tumor tissues compared to adjacent normal tissues. B. The survival rate for patients with high ANLN expression is worse than patients with low ANLN expression. C. ANLN was expressed in abundance in oral cancer cell lines. *P区 0.05 indicates significance. Each experiment was replicated at least three times. 
A

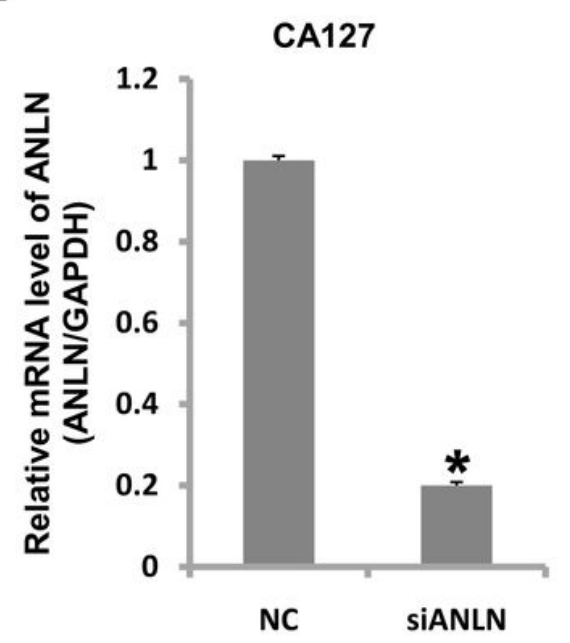

D

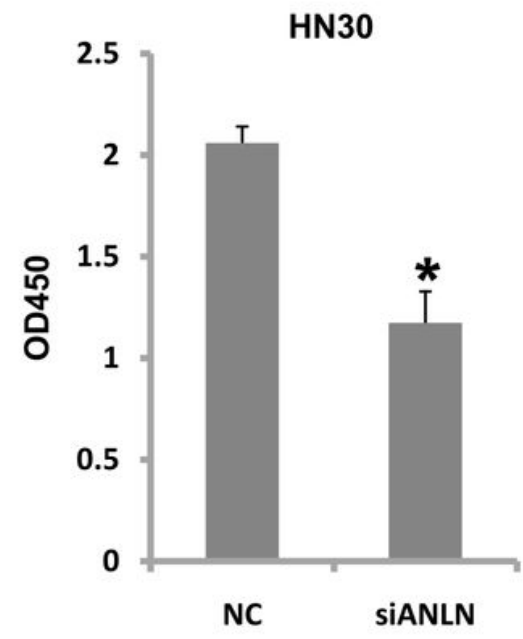

B

HN30

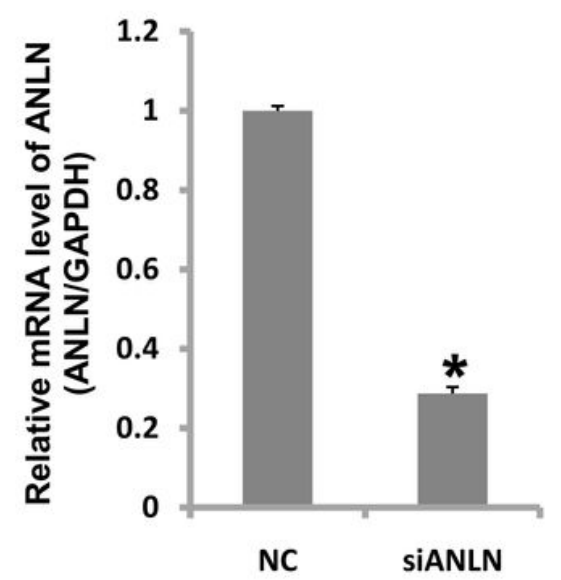

$\mathbf{E}$

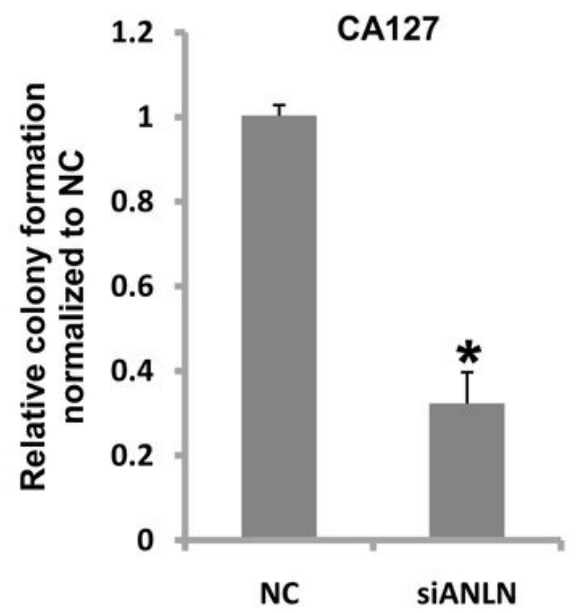

C

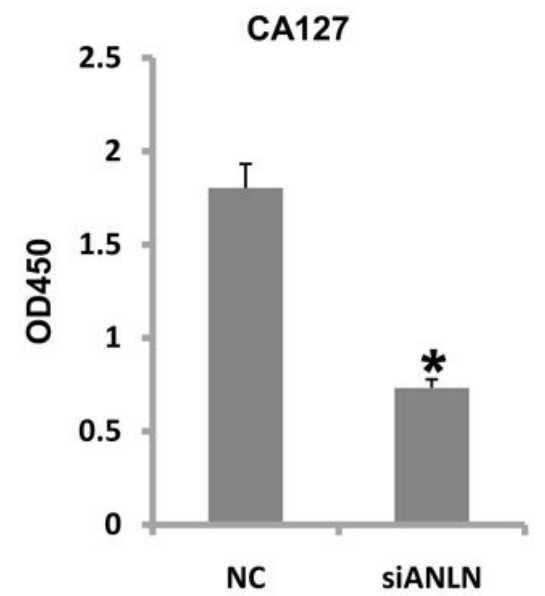

D

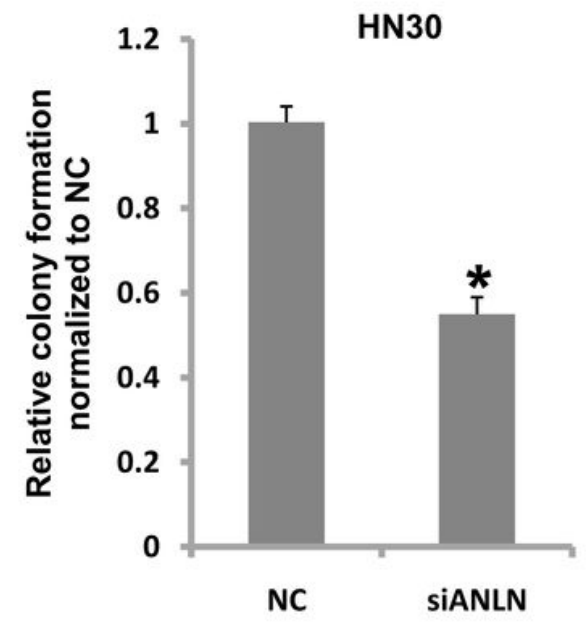

Figure 2

Knockdown of ANLN inhibited cell growth and proliferation in oral cancer. A. ANLN was knocked down efficiently in CAL27 cells. B. ANLN was knocked down efficiently in HN30 cells. C. Knockdown of ANLN inhibited cell proliferation in CAL27 cells. D. Knockdown of ANLN inhibited cell proliferation in HN30 cells. E. Knockdown of ANLN inhibited cell colony formation in CAL27 cells. F. Knockdown of ANLN inhibited cell colony formation in HN30 cells. ${ }^{*} \mathrm{P} \otimes 0.05$ indicates significance. Each experiment was replicated at least three times. 
A

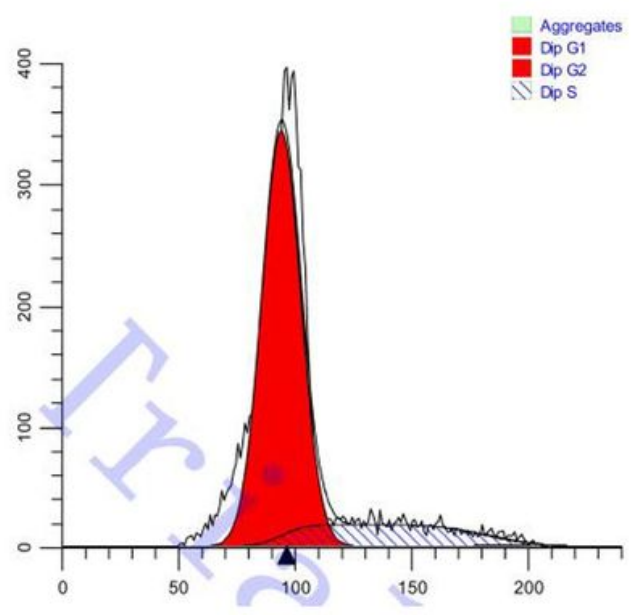

C

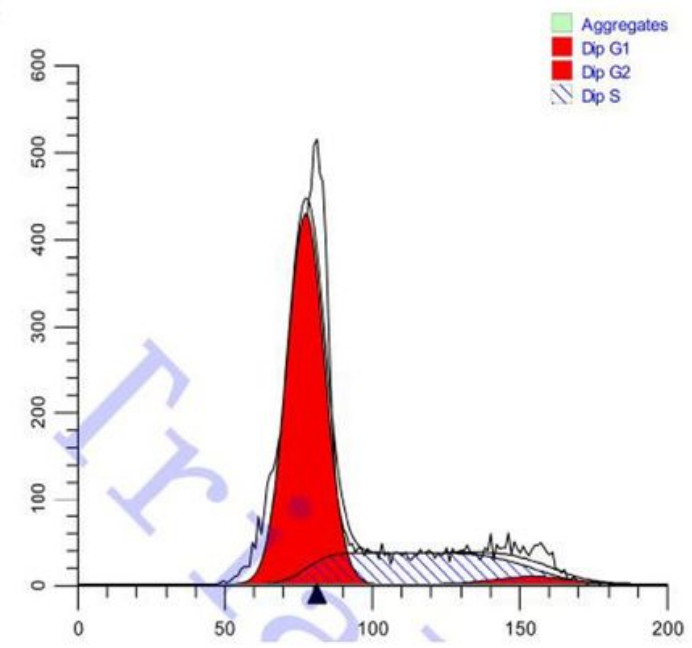

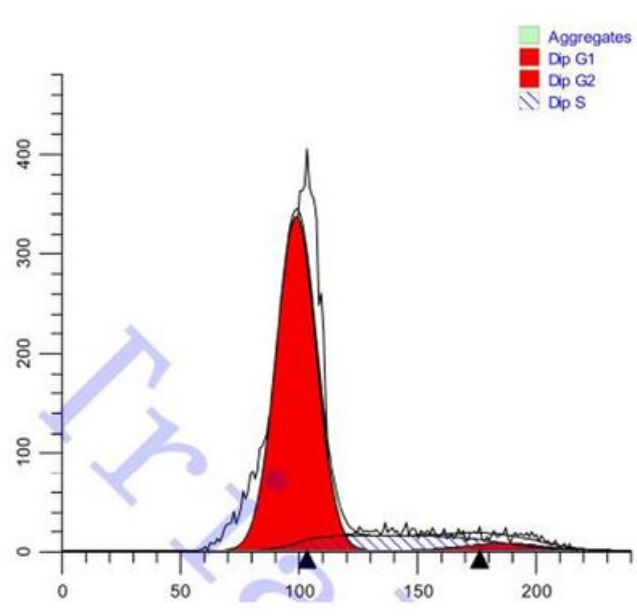

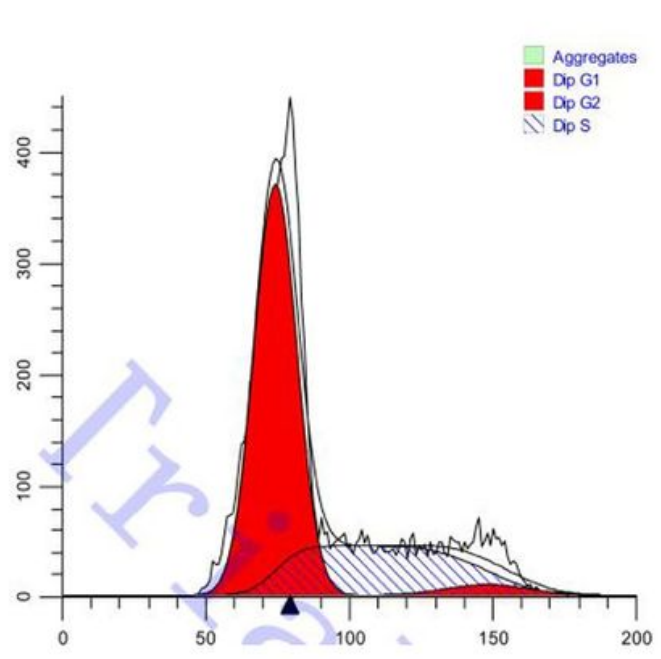

B

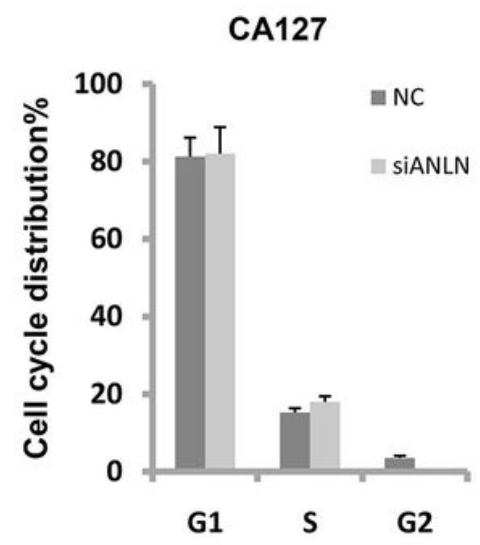

D

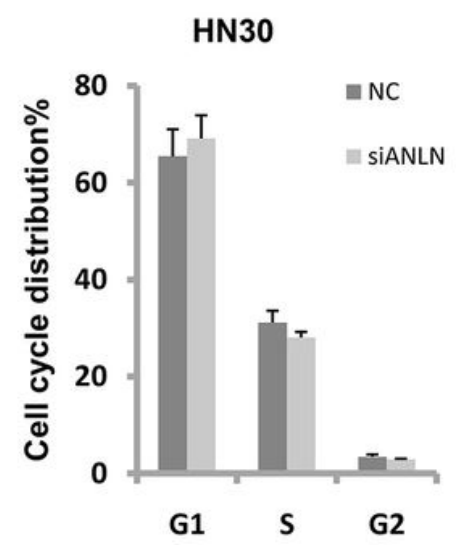

\section{Figure 3}

ANLN affected cell cycle transition in oral cancer. A. Cell cycle distribution in CAL27 cells after ANLN was reduced. B. Analysis of data in A. C. Cell cycle distribution in HN30 cells after ANLN was reduced. D. Analysis of data in C. Each experiment was replicated at least three times. 
A

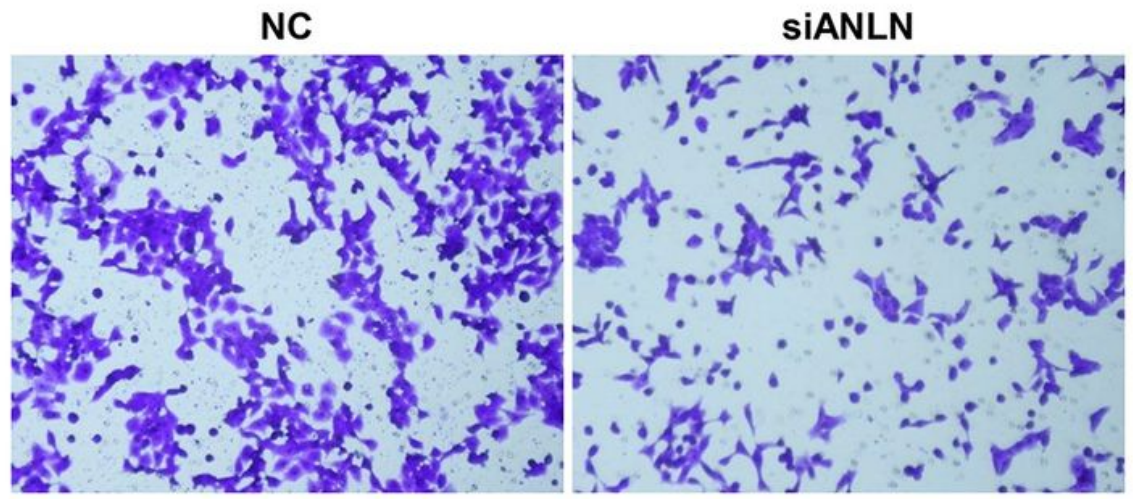

C

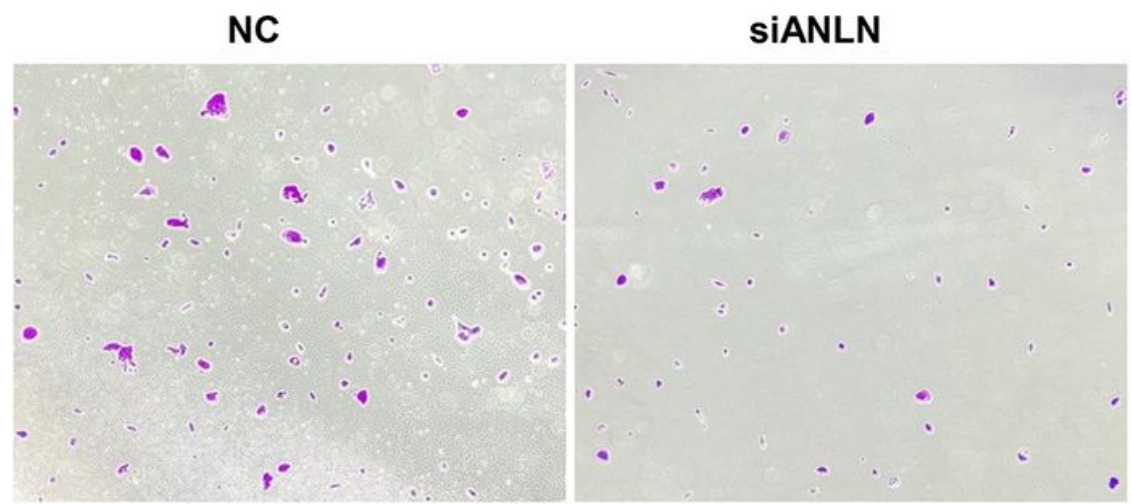

B

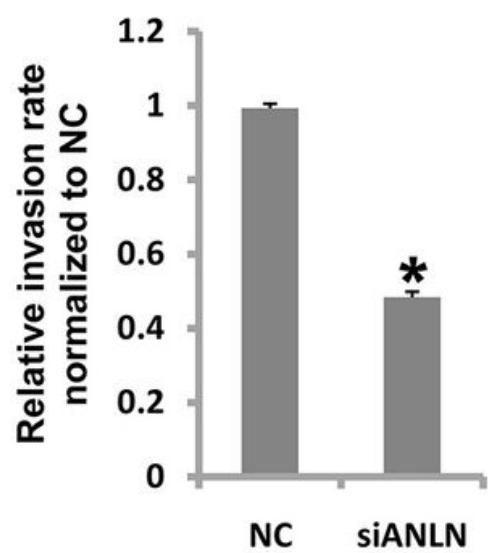

D

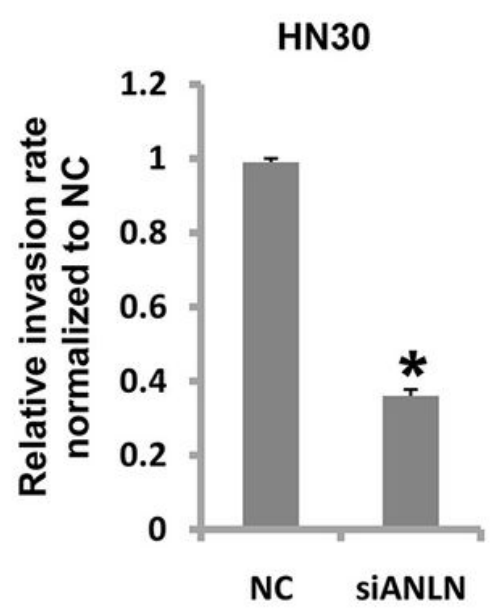

Figure 4

ANLN was essential to cell invasion in oral cancer. A. Cell invasion of CAL27 was suppressed after ANLN was reduced. B. Analysis of data in A. C. Cell invasion of HN30 was suppressed after ANLN was reduced. D. Analysis of data in C. ${ }^{*} \otimes 0.05$ indicates significance. Each experiment was replicated at least three times. 
A

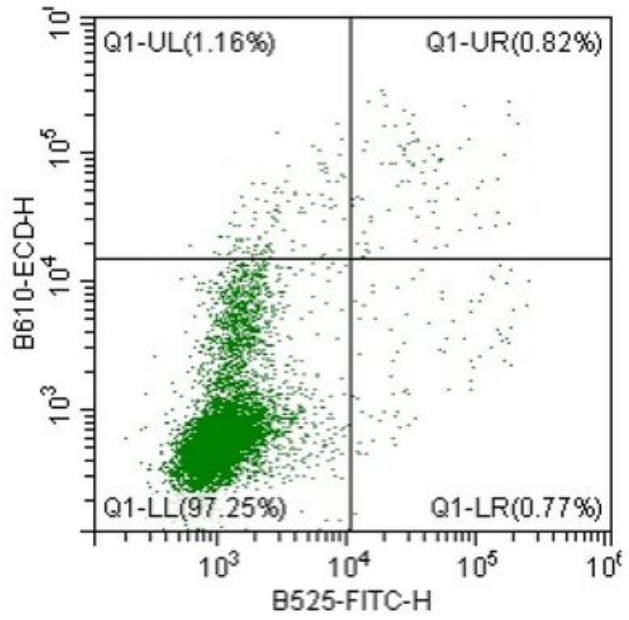

C

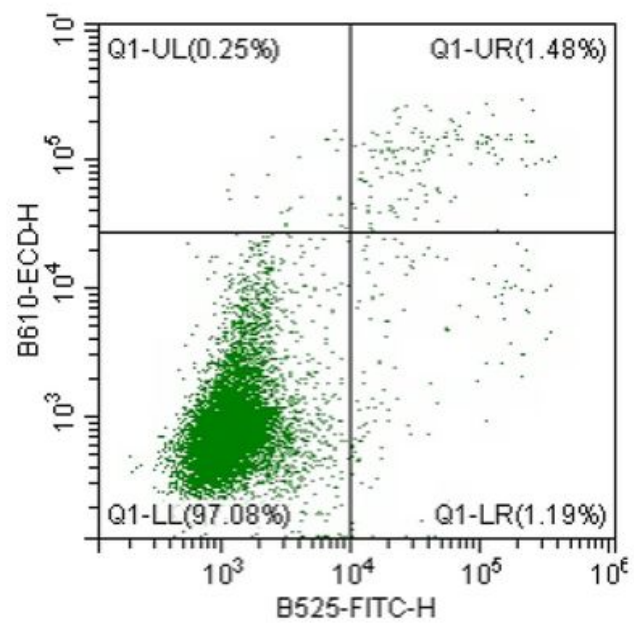

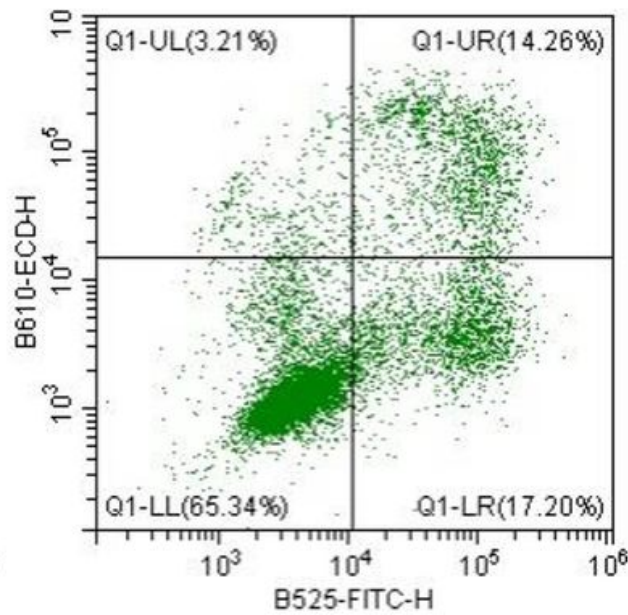

B

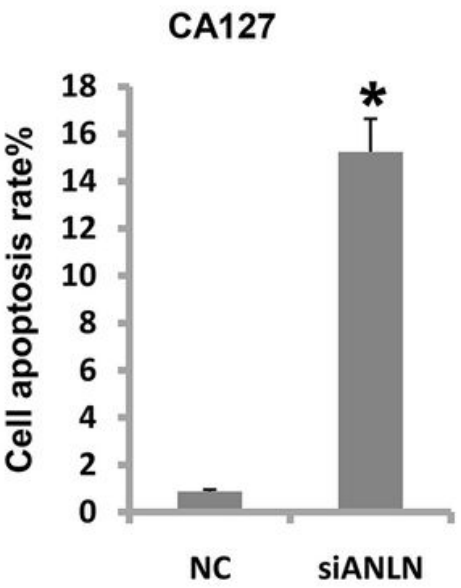

D

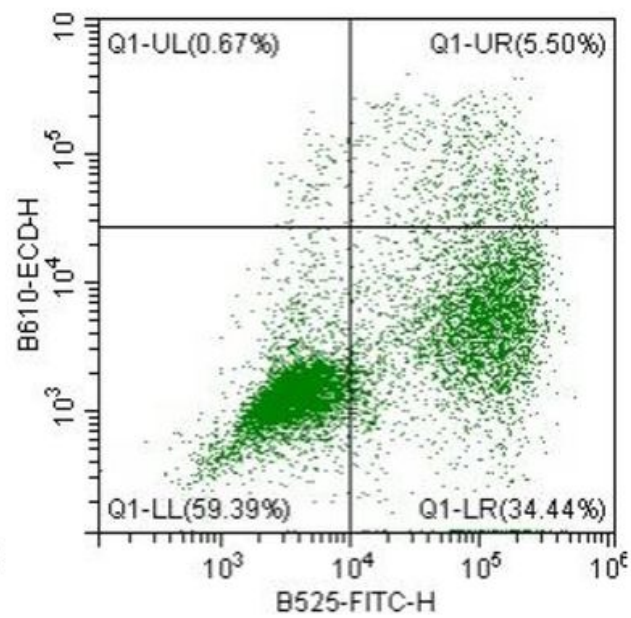

HN30

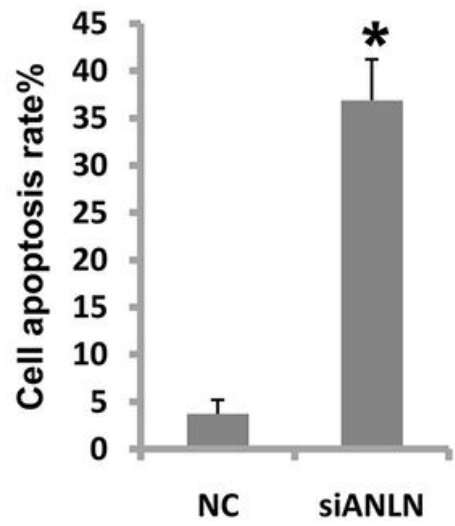

Figure 5

Knockdown of ANLN induced cell apoptosis in oral cancer. A. Cell apoptosis of CAL27 cells was induced after ANLN was reduced. B. Analysis of data in A. C. Cell apoptosis of HN30 cells was induced after ANLN was reduced. D. Analysis of data in C. *Pख0.05 indicates significance. Each experiment was replicated at least three times. 
A

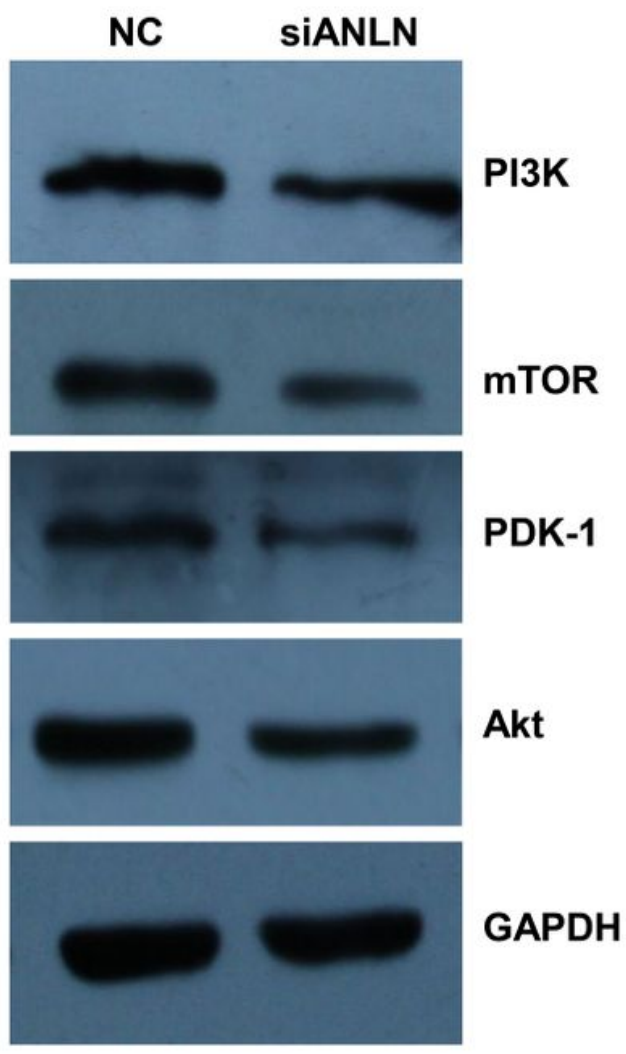

B

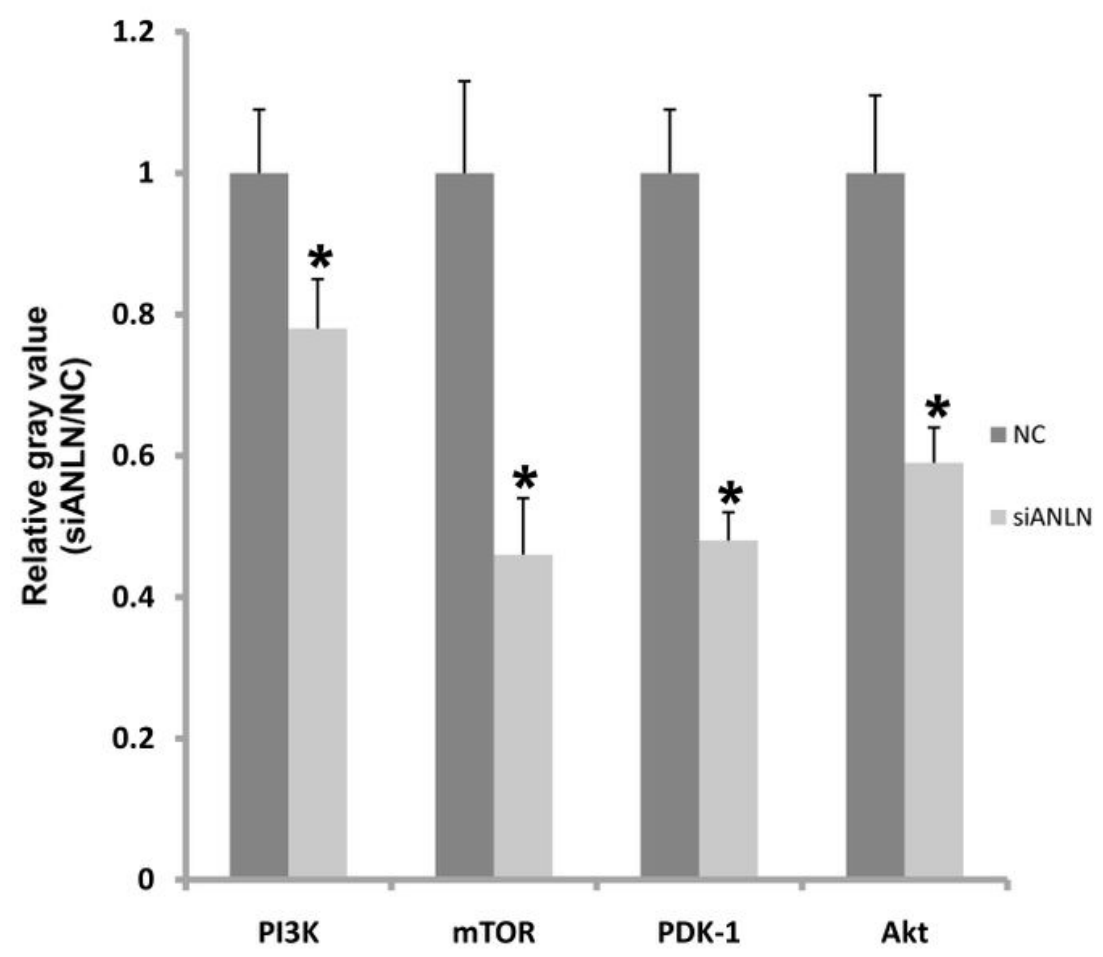

Figure 6

ANLN regulated activation of $\mathrm{PI} 3 \mathrm{~K} / \mathrm{mTOR}$ signaling pathway. A. Western blotting analysis showed that expression of PI3K, mTOR, PDK-1, and Akt was decreased after ANLN was reduced in CAL27 cells. B. Gray value analysis of data in A. *P囚0.05 indicates significance. Each experiment was replicated at least three times. 